\title{
A Generalization of the Folding Rule for the Clark-Kunen Semantics ${ }^{\star}$
}

\author{
Javier Álvez and Paqui Lucio \\ Basque Country University \\ \{javier.alvez, paqui.lucio\}@ehu.es
}

\begin{abstract}
In this paper, we propose more flexible applicability conditions for the folding rule that increase the power of existing unfold/fold systems for normal logic programs. Our generalized folding rule enables new transformation sequences that, in particular, are suitable for recursion introduction and local variable elimination. We provide some illustrative examples and give a detailed proof of correctness w.r.t. the Clark-Kunen semantics.
\end{abstract}

\section{Introduction}

Unfold/fold transformation systems were originally adapted by Tamaki and Sato in [28] to logic programming from the well-known Burstall-Darlington method for functional programming (see [6]). Tamaki and Sato's seminal unfold/fold system works on definite logic programs preserving their equivalence in the sense of the least Herbrand model. Since then, unfold/fold transformations of logic programs have been extensively studied and used (see [21] for a survey). In particular, different extensions of the Tamaki and Sato's system for dealing with negation have been proposed. The various semantics of negation in logic programming lead to different requirements in transformation rules depending on which semantics is intended to be preserved. The main motivation of this paper comes from our previous work (see $[18,1]$ ) in constructive negation (see [7]), which is sound and complete w.r.t. the Clark-Kunen semantics (see $[8,16]$ ). Hence, we are interested in transformation systems that preserve the Clark-Kunen semantics. The choice of the negation semantics is crucial for defining the side conditions of transformation rules. The following example illustrates this point.

Example 1. Given the following two clauses
$P_{0}$ :
1. $p \leftarrow q, r$
2. $q \leftarrow q$

by unfolding $q$ in the body of clause 1 with clause 2 we obtain clause 3 , which is a copy of the clause 1
$P_{1}$ :
3. $p \leftarrow q, r$
2. $q \leftarrow q$.

\footnotetext{
* This work has been partially supported by Spanish Project TIN2004-079250-C03-03.
} 
Now, if we allow to fold the body of the clause 3 using clause 1 (which can be seen as either a self-folding or a folding with a deleted clause) then we obtain

$$
\begin{array}{lll}
P_{2}: & \text { 4. } p \leftarrow p & \text { 2. } q \leftarrow q .
\end{array}
$$

First, note that the programs in the above example are propositional and positive, which makes more intrinsic the following problem. Let us consider another popular declarative semantics for negation: the well-founded semantics (see [13]). The programs $P_{0}$ and $P_{1}$ are equivalent w.r.t. both the Clark-Kunen and the well-founded semantics. Besides, $P_{0}$ and $P_{2}$ are equivalent w.r.t. the well-founded semantics, but they are not equivalent w.r.t. the Clark-Kunen semantics. More precisely, the well-founded model of $P_{0}$ and $P_{2}$ is $(\emptyset,\{p, q, r\})$, where no atom is true nor undefined and every atom is assigned to be false. However, Clark's completion of $P_{0}$ is

$$
(p \leftrightarrow(q \wedge r)) \wedge(q \leftrightarrow q) \wedge(r \leftrightarrow \text { false })
$$

so that $\neg p$ is a three-valued consequence of it, whereas $P_{2}$ 's completion is

$$
(p \leftrightarrow p) \wedge(q \leftrightarrow q) \wedge(r \leftrightarrow \text { false })
$$

and $\neg p$ is not a three-valued consequence of $P_{2}$ 's completion. Therefore, we need to provide extra-conditions to the folding rule in order to preserve the Clark-Kunen semantics, but these extra-conditions would be unnecessary if we considered the well-founded semantics.

There are many proposals for extending Tamaki and Sato's system (see [28]) for dealing with different semantic notions of negation. Seki (see [25]) showed that the system in [28] does not preserve finite-failure and introduced a modified folding rule that preserves finite failure and perfect model semantics in stratified normal programs. An extension of this system for general logic programs and for well-founded semantics was presented in [26]. The folding rule of [28] was also generalized in [14] to a simultaneous folding rule. Maher (see [20]) also extended the system to stratified general programs and the perfect model semantics. A more recent work on preserving stable and well-founded model semantics is [22]. ${ }^{1}$ In fact, as shown in Example 1, all these transformation systems do not preserve, in general, the Clark-Kunen semantics. Regarding the systems designed to preserve some completion-related semantics (see $[19,12,4]$ ), they enforce very rigid transformations. Indeed, they disable some useful transformations which do not spoil correctness w.r.t. the Clark-Kunen semantics, as illustrated in the next example.

Example 2. Given the following definition of a predicate $q$ such that $q\left(x_{1}, x_{2}\right)$ checks whether the list $x_{1}$ is not a sublist of $x_{2}$

1. $q\left(x_{1}, x_{2}\right) \leftarrow$ member $\left(y, x_{1}\right), \neg$ member $\left(y, x_{2}\right)$

2. member $\left(v,\left[\left.v\right|_{-}\right]\right) \leftarrow$

3. member $\left(v_{1},\left[-\mid v_{2}\right]\right) \leftarrow \operatorname{member}\left(v_{1}, v_{2}\right)$.

\footnotetext{
${ }^{1}$ It is well known that the well-founded model is one of the stable models, which is minimal in some sense.
} 
First, we unfold member $\left(y, x_{1}\right)$ in clause 1 w.r.t. clauses 2 and 3

4. $\quad q\left(\left[z_{1} \mid-\right], z_{2}\right) \leftarrow \neg$ member $\left(z_{1}, z_{2}\right)$

5. $\quad q\left(\left[-\mid z_{1}\right], z_{2}\right) \leftarrow \operatorname{member}\left(w, z_{1}\right), \neg \operatorname{member}\left(w, z_{2}\right)$.

Then, we fold clause 5 using clause 1 , which has been removed in the previous step. The resulting definition of $q$ is

4. $\quad q\left(\left[z_{1} \mid-\right], z_{2}\right) \leftarrow \neg$ member $\left(z_{1}, z_{2}\right)$

6. $\quad q\left(\left[-\mid z_{1}\right], z_{2}\right) \leftarrow q\left(z_{1}, z_{2}\right)$.

The transformation in Example 2 is forbidden in all the existing systems which consider completion-related semantics, in spite of the fact that it is correct w.r.t. the Clark-Kunen semantics. For example, the so-called reversible folding requires the folded and folder clauses to be in the current program. This is the folding used in $[19,12]$. In the above Example 2, the folder clause is not in the current program, hence the systems in [19,12] cannot be used. In [4], folding is allowed through the use of semantic conditions if the folded clause comes from the folder one, which has to be non-recursive, and all the literals to be folded have been obtained by unfolding. In Example 2, the literal ${ }^{2} \neg \operatorname{member}\left(w, z_{2}\right)$ is inherited from the original program, thus it is not the result of an unfolding step. Other systems split the predicates into new/old predicates, where the old predicates cannot depend on the new predicates and the new predicates are nonrecursive. This is case in the previously cited system in [25] where the following two conditions are required:

(1) only the clauses with a new predicate in its head can be used as folder clauses, and

(2) the predicate in the head of the folded clause is an old predicate or all the literals to be folded are the result of a previous unfolding.

In Example 2, the predicate in the head of the folder and folded clause is the same (that is, the predicate $q$ ), thus we cannot use the system in [25] since $\neg$ member $\left(w, z_{2}\right)$ is inherited from the original program. The four-step transformation schema proposed in [5] uses the same partition of predicates, and, once again, when the predicate in the head of the folded clause is new, all the literals to be folded have to be the result of an unfolding, therefore this system cannot be used in Example 2. Finally, the folding rule in the system proposed in [23] for first-order general programs, which also uses the new/old partition, ${ }^{3}$ requires the same condition.

In this paper, we introduce a transformation system for normal logic programs that preserves the Clark-Kunen semantics and is more flexible than the existing ones with the following two advantages:

1. the folder clause can be taken from any program in the transformation sequence.

\footnotetext{
${ }^{2}$ The negative character of the literal is not relevant for this discussion.

${ }^{3}$ By contrast, the new predicates can be recursive in this proposal.
} 
2. the folded literals do not necessarily come from unfolding.

Outline of the paper. In the next section, Section 2, which is split in three subsections, we establish the notations and describe necessary results on semantics of logic programs and unfold/fold systems. Section 3 is devoted to defining new conditions for the folding rule, where we motivate the problem using some examples and we then prove the correctness of the resulting system. In Section 4 , we give some concluding remarks and indicate some of the open problems which need to be solved.

\section{Preliminaries}

We assume that the reader is familiar with the basic concepts of logic programming. Throughout the paper we use the standard terminology of [17] and [2]. In particular, we will use the standard notions of substitution of variables by terms, unifier and most general unifier (briefly mgu). A bar is used to abbreviate tuples of objects. For example, $\bar{x}$ denotes a tuple of variables $x_{1}, \ldots, x_{n}$, the tuple of literals $L_{1}, \ldots, L_{n}$ is denoted by $\bar{L}$ and the substitution $\sigma=\left\{x_{1} \leftarrow t_{1}, \ldots, x_{n} \leftarrow\right.$ $\left.t_{n}\right\}$ is abbreviated as $\{\bar{x} \leftarrow \bar{t}\}$. Besides, $\sigma$ is sometimes interpreted as the conjunction of equations $x_{1} \approx t_{1} \wedge \ldots \wedge x_{n} \approx t_{n}$ (abbreviated as $\bar{x} \approx \bar{t}$ ), and hence $\neg \sigma$ is interpreted as the disjunction of disequations $x_{1} \not \approx t_{1} \vee \ldots \vee x_{n} \not \approx t_{n}$ (abbreviated as $\bar{x} \not \approx \bar{t}$ ).

We consider (normal) logic programs which are finite sequences (not sets) of normal clauses of the form $A \leftarrow \bar{L}$. Throughout this work, programs are given modulo reordering of the literals in bodies and standardization apart is always assumed.

The definition of the atom $L$ in a program $P$, denoted by $\operatorname{Def}_{P}[L]$, is the sequence of clauses from $P$ such that its clause head unifies with $L$. If $L$ is a flat atom on the predicate $p$, then we also say that $\operatorname{Def}_{P}[L]$ is the definition of the predicate $p$.

An atom $L$ directly depends on the atom $N$ in a program $P$ iff there exists a clause $(H \leftarrow \bar{B}) \in \operatorname{Def}_{P}[L]$ such that $N \in \bar{B}$. Besides, $L$ is also said to directly depend on every clause in $\operatorname{Def}_{P}[L]$. The dependence relation on atoms/clauses is given by the reflexive and transitive closure of the directly dependence relation.

\subsection{The Clark-Kunen Semantics and Non-failure}

In [8], Clark proposed the following to complete the definition of predicates. Supposing that $\operatorname{Def}_{P}[p(\bar{x})]$ consists of the following $m$ clauses $\left\langle p\left(\bar{t}^{k}\right) \leftarrow \bar{B}^{k}\right| 1 \leq$ $k \leq m\rangle$, the completion formula of a predicate $p \in \operatorname{Pred}_{\mathcal{L}}(P)$ is

$$
\left(p(\bar{x}) \leftrightarrow \bigvee_{k=1}^{m} \exists \bar{z}^{k}\left(\bar{x} \approx \bar{t}^{k} \wedge \bar{B}^{k}\right)\right)^{\forall}
$$

where $\bar{z}^{k}=\operatorname{Var}\left(\bar{t}^{k} \cdot \bar{B}^{k}\right)$ for each $1 \leq k \leq m$. If $m=0$, then it is equivalent to $(p(\bar{x}) \leftrightarrow \text { false })^{\forall}$. The Clark completion of a program $P$, denoted by 
$\operatorname{Comp}(P)$, consists of the conjunction of the completion formulas of each predicate $p \in \operatorname{Pred}_{\mathcal{L}}(P)$ and the axioms of the free equality theory $\mathrm{FET}_{\mathcal{L}}$ (see [9]). Whenever the definition of $p$ is free of local variables, the negation of (1)

$$
\left(\neg p(\bar{x}) \leftrightarrow \bigwedge_{k=1}^{m} \forall \bar{z}^{k}\left(\bar{x} \not \bar{t}^{k} \vee \neg \bar{B}^{k}\right)\right)^{\forall}
$$

can be transformed (see $[24,3])$ into a logically equivalent formula of the form

$$
\left(\neg p(\bar{x}) \leftrightarrow \bigvee_{h=1}^{n} \exists \bar{w}^{h}\left(\bar{x} \approx \bar{s}^{h} \wedge \bar{M}^{h}\right)\right)^{\forall} .
$$

From this formula, we obtain a finite sequence of expressions

$$
\left\langle\neg p\left(\bar{s}^{h}\right) \leftarrow \bar{M}^{h} \mid 1 \leq h \leq n\right\rangle
$$

which yields $\operatorname{Def}_{P}[\neg p(\bar{x})]$. Otherwise, if some clause in $\operatorname{Def}_{P}[p(\bar{x})]$ contains local variables, then we consider that $\operatorname{Def}_{P}[\neg p(\bar{x})]$ is undefined, i.e. $\neg p(\bar{x})$ has no definition. Once we have a definition for negative literals, the dependence relation is extended to negative literals in the natural way.

Example 3. Let us consider the following definition of the predicate member

1. member $\left(v,\left[\left.v\right|_{-}\right]\right) \leftarrow$

2. member $\left(v_{1},\left[-\mid v_{2}\right]\right) \leftarrow$ member $\left(v_{1}, v_{2}\right)$.

The completion formula of member is

$$
\begin{aligned}
\left(\operatorname{member}\left(x_{1}, x_{2}\right) \leftrightarrow\right. & \exists v, v^{\prime}\left(x_{1} \approx v \wedge x_{2} \approx\left[v \mid v^{\prime}\right]\right) \vee \\
& \left.\exists v_{1}, v_{2}, v^{\prime \prime}\left(x_{1} \approx v_{1} \wedge x_{2} \approx\left[v^{\prime \prime} \mid v_{2}\right] \wedge \operatorname{member}\left(v_{1}, v_{2}\right)\right)\right)^{\forall} .
\end{aligned}
$$

From the above formula, we obtain

$$
\begin{aligned}
\left(\neg \text { member }\left(x_{1}, x_{2}\right) \leftrightarrow\right. & \forall v, v^{\prime}\left(x_{1} \not v \vee x_{2} \not \approx\left[v \mid v^{\prime}\right]\right) \wedge \\
& \forall v_{1}, v_{2}, v^{\prime \prime}\left(x_{1} \not v_{1} \vee x_{2} \not \approx\left[v^{\prime \prime} \mid v_{2}\right] \vee \neg \operatorname{member}\left(v_{1}, v_{2}\right)\right) .
\end{aligned}
$$

Refining the right-hand subformula, we get

$$
\begin{aligned}
\left(\neg \text { member }\left(x_{1}, x_{2}\right) \leftrightarrow\right. & {\left[\forall v^{\prime}\left(x_{2} \not \approx\left[x_{1} \mid v^{\prime}\right]\right) \wedge \forall v_{2}, v^{\prime \prime}\left(x_{2} \not \approx\left[v^{\prime \prime} \mid v_{2}\right]\right)\right] \vee } \\
& {\left[\forall v^{\prime}\left(x_{2} \not \approx\left[x_{1} \mid v^{\prime}\right]\right) \wedge\right.} \\
& \exists v_{1}, v_{2}, v^{\prime \prime}\left(x_{1} \approx v_{1} \wedge x_{2} \approx\left[v^{\prime \prime} \mid v_{2}\right] \wedge \neg \operatorname{member}\left(v_{1}, v_{2}\right)\right)
\end{aligned}
$$

which is, after simplification, equivalent to

$$
\begin{aligned}
\left(\neg \operatorname{member}\left(x_{1}, x_{2}\right) \leftrightarrow\right. & \exists z_{1}, z_{2}\left(x_{1} \approx z_{1} \wedge x_{2} \approx z_{2} \wedge \forall y_{1}, y_{2}\left(z_{2} \not \approx\left[y_{1} \mid y_{2}\right]\right)\right) \vee \\
& \exists z_{1}, z_{2}, z_{3}\left(x_{1} \approx z_{1} \wedge x_{2} \approx\left[z_{2} \mid z_{3}\right] \wedge z_{1} \not \approx z_{2} \wedge\right. \\
& \left.\neg \operatorname{member}\left(z_{1}, z_{3}\right)\right) .
\end{aligned}
$$

Since the second argument of member is a list from the last formula we can obtain the following normal ${ }^{4}$ clauses that define $\neg$ member

\footnotetext{
${ }^{4} w_{1} \not w_{2}$ is a negative literal since $\approx$ should be defined by the single clause $x \approx x \leftarrow$
} 
3. $\neg$ member $\left(_{-},[]\right) \leftarrow$

4. $\neg$ member $\left(w_{1},\left[w_{2} \mid w_{3}\right]\right) \leftarrow w_{1} \not \approx w_{2}, \neg \operatorname{member}\left(w_{1}, w_{3}\right)$.

In this work, the semantics given to a program is the Clark-Kunen semantics as proposed in [16]; that is, the three-valued logical consequence of the Clark completion. Following [21], the Clark-Kunen semantics of a program $P$ is defined by

$$
\operatorname{COMP}[P, \leftarrow \bar{L}]=\left\{c \mid \operatorname{Comp}(P) \models_{3}(\bar{L} \wedge c)^{\forall}\right\}
$$

where $\leftarrow \bar{L}$ is a goal, $c$ is a general equality constraint and $\models_{3}$ stands for the three-valued logical consequence relation, as defined in [16]. Regarding equivalence of programs, we consider that two programs $P_{1}$ and $P_{2}$ are equivalent, denoted by $P_{1} \equiv P_{2}$, iff the set of logical consequences of $\operatorname{Comp}\left(P_{1}\right)$ and $\operatorname{Comp}\left(P_{2}\right)$ are identical.

Definition 1. Given two programs $P_{1}$ and $P_{2}$,

(i) $P_{1} \preceq P_{2}$ iff $\operatorname{COMP}\left[P_{1}, \leftarrow \bar{L}\right] \subseteq \operatorname{COMP}\left[P_{2}, \leftarrow \bar{L}\right]$ for any goal $\leftarrow \bar{L}$.

(ii) $P_{1} \equiv P_{2}$ iff $P_{1} \preceq P_{2}$ and $P_{2} \preceq P_{1}$.

A desirable property of a semantic notion is relevance, which is defined in [11] and extensively used in [21]. Intuitively, a semantics is relevant iff the semantic value of any goal $\leftarrow \bar{L}$ w.r.t. a program $P$ is exactly given by the clauses on which the literals in $\bar{L}$ depend. In the absence of relevance, some transformation rules, such as new definition and deletion, are not trivially correct (see [21]). As defined above, the Clark-Kunen semantics is relevant. However, by changing $\models_{3}$ by the classical bi-valued logical consequence notion (as in [21]), relevance is lost.

The so-called Shepherdson operators $\mathrm{T}$ and $\mathrm{F}$, which were introduced in [27], give a very useful characterization of the Clark-Kunen semantics. These operators provide a bottom-up scheme for computing the success- and fail-answers of any literal by means of equality constraints. In the next definition, we adapt the original definition of the operators in [27] to our purposes.

Definition 2 ([27]). Let $P$ be a program and $L$ a literal such that $\operatorname{Def}_{P}[L]=$ $\left\langle H_{k} \leftarrow \bar{B}^{k} \mid 1 \leq k \leq m\right\rangle$. The operators $\mathrm{T}$ and $\mathrm{F}$ are inductively defined as

$$
\begin{array}{ll}
\mathrm{T}_{0}^{P}[L]=\text { false } & \mathrm{F}_{0}^{P}[L]=\text { false } \\
\mathrm{T}_{n+1}^{P}[L]=\bigvee_{k=1}^{m} \exists \bar{x}^{k}\left(\mathrm{~T}_{n}^{P}\left[\bar{B}^{k} \theta^{k}\right]\right) & \mathrm{F}_{n+1}^{P}[L]=\bigwedge_{k=1}^{m} \forall \bar{x}^{k}\left(\mathrm{~F}_{n}^{P}\left[\bar{B}^{k} \theta_{k}\right]\right)
\end{array}
$$

where $\theta_{k}=m g u\left(L, H_{k}\right)$ and $\bar{x}^{k}=\operatorname{Var}\left(H_{k} \cdot \bar{B}^{k}\right)$ for $1 \leq k \leq m$. Besides, the extension to constants and connectives is

$$
\begin{aligned}
& \mathrm{T}_{n}^{P}[\text { true }]=\text { true } \quad \mathrm{T}_{n}^{P}[\bar{L} \theta] \quad=\mathrm{T}_{n}^{P}[\bar{L}] \wedge \theta \\
& \mathrm{F}_{n}^{P}[\text { true }]=\text { false } \quad \mathrm{F}_{n}^{P}[\bar{L} \theta] \quad=\mathrm{F}_{n}^{P}[\bar{L}] \vee \neg \theta \\
& \mathrm{T}_{n}^{P}[\neg \bar{L}]=\mathrm{F}_{n}^{P}[\bar{L}] \quad \mathrm{T}_{n}^{P}[\bar{M} \wedge \bar{N}]=\mathrm{T}_{n}^{P}[\bar{M}] \wedge \mathrm{T}_{n}^{P}[\bar{N}] \\
& \mathrm{F}_{n}^{P}[\neg \bar{L}]=\mathrm{T}_{n}^{P}[\bar{L}] \quad \mathrm{F}_{n}^{P}[\bar{M} \wedge \bar{N}]=\mathrm{F}_{n}^{P}[\bar{M}] \vee \mathrm{F}_{n}^{P}[\bar{N}]
\end{aligned}
$$


for any $n \in \mathbb{N}$, every substitution $\theta$ and every tuple of literals $\bar{L}, \bar{M}$ and $\bar{N}$.

It is easy to see that $\mathrm{T}_{n}^{P}[L] / \mathrm{F}_{n}^{P}[L]$ represents the successes/failures of $L$ that can be derived from $P$ in $n$ steps. In particular, facts produce the one level successes.

Example 4. Let $\mathcal{F}_{\mathcal{L}}=\left\{0_{/ 0}, s_{/ 1}\right\}$ and $P$ be the following program

1. $p(0) \leftarrow$

2. $p(s(s(x))) \leftarrow p(x)$.

We obtain the following constraints from the Shepherdson operators

$$
\begin{array}{ll}
\mathrm{T}_{1}^{P}[p(x)]=(x \approx 0) & \mathrm{F}_{1}^{P}[p(x)]=(x \not \approx 0 \wedge \forall v(x \not \approx s(s(v)))) \\
\mathrm{T}_{2}^{P}[p(x)]=(x \approx 0 \vee x \approx s(s(0))) & \mathrm{F}_{1}^{P}[p(s(x))]=\forall v(x \not \approx s(v)) .
\end{array}
$$

The operators $\mathrm{T}$ and $\mathrm{F}$ are dual and, hence, they satisfy dual properties. Moreover, the proofs for both operators also follow dual steps and, since the operator $\mathrm{T}$ is existentially quantified, the proofs for $\mathrm{T}$ are usually easier than for $\mathrm{F}$. Thus, we formulate properties for both operators using the notation $0 \in\{\mathrm{T}, \mathrm{F}\}$, but only give proofs for the operator $\mathrm{F}$, because they can be easily adapted to $\mathrm{T}$.

An easy consequence of Definition 2 is the following monotonicity property.

Proposition 1. For any program $P$, literal $L, n \in \mathbb{N}$ and $0 \in\{\mathrm{T}, \mathrm{F}\}$,

$$
\operatorname{FET}_{\mathcal{L}} \models\left(\mathrm{O}_{n}^{P}[L] \rightarrow \mathrm{O}_{n+1}^{P}[L]\right)^{\forall} .
$$

The characterization of the Clark-Kunen semantics by the Shepherdson operators directly follows from Lemma 4.1 in [27] and Theorem 6.3 in [16], and can be stated as

Theorem $1([\mathbf{1 6}, \mathbf{2 7}])$. For any normal program $P$ and any goal $\bar{L}$,

$$
\theta \in \operatorname{COMP}[P, \bar{L}] \Longleftrightarrow \text { there exists some } n \in \mathbb{N} \text { such that } \operatorname{FET}_{\mathcal{L}} \models \mathrm{T}_{n}^{P}[\bar{L} \theta] .
$$

By means of this characterization, we obtain the following result that is very useful for proving equivalences between programs in the rest of this paper.

Corollary 1. Let $P_{1}, P_{2}$ be two programs. $P_{1} \preceq P_{2}$ iff for all $n_{1} \in \mathbb{N}, p \in$ $\left.\operatorname{Pred}_{\mathcal{L}}\left(P_{1}\right) \cap \operatorname{Pred}_{\mathcal{L}}\left(P_{2}\right)\right)$ and $\mathrm{O} \in\{\mathrm{T}, \mathrm{F}\}$, there exists some $n_{2} \in \mathbb{N}$ such that

$$
\operatorname{FET}_{\mathcal{L}} \models_{3}\left(\mathrm{O}_{n_{1}}^{P_{1}}[p(\bar{x})] \rightarrow \mathrm{O}_{n_{2}}^{P_{2}}[p(\bar{x})]\right)^{\forall} .
$$

Finally, we define the class of goals that do not fail on some variables.

Definition 3. Let $P$ be a program, $\leftarrow \bar{L}$ a goal and $\bar{x} \subseteq \operatorname{Var}(\bar{L})$. The goal $\leftarrow \bar{L}$ is non-failing on $\bar{x}$ w.r.t. $P$ iff for all $n \in \mathbb{N}$

$$
\mathrm{FET}_{\mathcal{L}} \models \forall \bar{x} \exists \bar{y} \neg \mathrm{F}_{n}^{P}[\bar{L}]
$$

where $\bar{y}=\operatorname{Var}(\bar{L}) \backslash \bar{x}$. 
Next, we illustrate the notion of non-failing goals with two examples.

Example 5. Let us consider the following program

1. $\operatorname{add}(0, n, n)$

2. $\operatorname{add}\left(s\left(n_{1}\right), n_{2}, s\left(n_{3}\right)\right) \leftarrow \operatorname{add}\left(n_{1}, n_{2}, n_{3}\right)$.

The literal $\operatorname{add}\left(x_{1}, x_{2}, x_{3}\right)$ is non-failing on the variables $\left\{x_{1}, x_{2}\right\}$. However, the literal add $\left(x_{1}, x_{2}, x_{3}\right)$ is failing on $\left\{x_{2}, x_{3}\right\}$.

Example 6. Let us consider the following program

1. $\operatorname{ack}(0, n, s(n))$

2. $\operatorname{ack}\left(s\left(n_{1}\right), 0, n_{2}\right) \leftarrow \operatorname{ack}\left(n_{1}, s(0), n_{2}\right)$

3. $\operatorname{ack}\left(s\left(n_{1}\right), s\left(n_{2}\right), n_{3}\right) \leftarrow \operatorname{ack}\left(s\left(n_{1}\right), n_{2}, y\right), \operatorname{ack}\left(n_{1}, y, n_{3}\right)$.

The goal $\operatorname{ack}\left(s\left(x_{1}\right), x_{2}, v\right), \operatorname{ack}\left(x_{1}, v, x_{3}\right)$ is non-failing on $\left\{x_{3}\right\}$.

The interested reader is referred to [10] for details on algorithms that decide if a goal is non-failing. Roughly speaking, given a goal $G$ and a set $\bar{x}$ of its variables, the algorithm checks whether the set of constraints associated to all the non-failing goals that can be obtained by resolution from $G$ covers all the possible values for $\bar{x}$. According to [10], the covering problem is co-NP-hard.

\subsection{Unfold-fold Transformation Systems}

In this section, we recall the classical unfold/fold transformation rules that were introduced in [28], adapting them to our notation. We also provide some wellknown correctness results that we will use later.

A sequence of programs $\left\langle P_{0}, \ldots, P_{n}\right\rangle$ is a transformation sequence if for each $1 \leq i \leq n, P_{i}$ is the result of transforming $P_{i-1}$ using some rule. Besides, $\left\langle P_{0}, \ldots, P_{n}\right\rangle$ is correct if $P_{0}$ and $P_{i}$ are equivalent for every $1 \leq i \leq n$. By extension, a transformation rule is said to be correct if it preserves equivalence.

Program transformation systems usually work with some information related to the transformation process itself. For example, in à la Tamaki-Sato systems (see $[26,28])$, the clauses that are obtained after unfolding are marked "foldable". In [23], literals (instead of clauses) are marked "foldable". In other systems (see [15]), counters of unfolding/folding steps are associated with clauses in order to both formulate folding applicability conditions and to characterize the improvement of execution. In this paper, we associate two natural numbers $\left\langle L_{\text {unf }}, L_{\mathrm{fld}}\right\rangle$ with each body literal $L$, called unfolding and folding time-stamps. A time-stamp $L_{\text {unf }} / L_{\mathrm{fld}}$ is either zero or the index $i$ of the program $P_{i}$ in the transformation sequence $\left\langle P_{0}, \ldots, P_{n}\right\rangle$ in which $L$ is obtained by unfolding/folding. Hence, in the initial program $P_{0}$, all time-stamp are zero and they are appropriately updated at each transformation step.

Before recalling the usual rules in unfold/fold systems, let us fix the following conventions that we will use in the formulation of the transformation rules:

(1) we always refer to a transformation sequence $\left\langle P_{0}, \ldots, P_{i}\right\rangle$, 
(2) $P_{i+1}$ is the next program obtained by the transformation from $P_{i}$, and

(3) if a clause $C$ has not been transformed from $P_{i}$ to $P_{i+1}$, then the time-stamps for the literals in $C$ are equal in both programs.

Next, we re-formulate unfold/fold systems incorporating time-stamps issues.

Rule 1 New Definition. If $p \notin \bigcup_{j=0}^{i} \operatorname{Pred}_{\mathcal{L}}\left(P_{j}\right)$ and $S=\left\langle C_{1}, \ldots, C_{m}\right\rangle$ is a definition of the predicate $p$ such that $\operatorname{Pred}_{\mathcal{L}}(S) \subseteq\left(\operatorname{Pred}_{\mathcal{L}}\left(P_{i}\right) \cup\{p\}\right)$, then $P_{i+1}=P_{i} \cup S$. The pair of time-stamps $\left\langle L_{\mathrm{unf}}, L_{\mathrm{fld}}\right\rangle$ is $\langle 0,0\rangle$ for every literal $L$ occurring in the body of any clause from $S$.

Rule 2 Unfolding. If $C=H \leftarrow \bar{M}, L$ is a clause in $P_{i}$ (unfolded clause) and $\operatorname{Def}_{P_{j}}[L]=\left\langle L_{k} \leftarrow \bar{N}^{k} \mid 1 \leq k \leq m\right\rangle$ for some $0 \leq j \leq i$, then $P_{i+1}=$ $\left(P_{i} \backslash C\right) \cup\left\langle\left(H \leftarrow \bar{M}, \bar{N}^{k}\right) \theta_{k} \mid 1 \leq k \leq m\right\rangle$ where $\theta_{k}=m g u\left(L, L_{k}\right)$ for every $1 \leq k \leq m$. For every clause $\left(H \leftarrow \bar{M}, \bar{N}^{k}\right) \theta_{k}$ in the program $P_{i+1}$, the pair of time-stamps $\left\langle N_{\text {unf }}^{\prime}, N_{\text {fld }}^{\prime}\right\rangle$ is $\left\langle i+1, L_{\mathrm{fld}}\right\rangle$ for each literal $N^{\prime} \in \bar{N}^{k} \theta_{k}$ and, besides, the pair $\left\langle M_{\mathrm{unf}}^{\prime}, M_{\mathrm{fld}}^{\prime}\right\rangle$ is equal to $\left\langle M_{\mathrm{unf}}, M_{\mathrm{fld}}\right\rangle$ in $P_{i}$ for each $M^{\prime}=M \theta_{k} \in \bar{M} \theta_{k}$.

If $P_{i}=P_{j}$ and the unfolded clause $(H \leftarrow \bar{M}, L) \in \operatorname{Def}_{P_{j}}[L]$, then the Unfolding transformation is said to be a self-unfolding.

Rule 3 Folding. If $H \leftarrow \bar{M}, \bar{N}$ is a clause in $P_{i}$ (folded clause), $L \leftarrow \bar{N}^{\prime}$ is a clause in $P_{j}$ (folder clause) for some $0 \leq j \leq i$ and $\sigma$ is a substitution such that

(a) domain $(\sigma)=\operatorname{Var}(L)$,

(b) $H \leftarrow \bar{M}, \bar{N}$ and $H \leftarrow \bar{M}, \bar{N}^{\prime} \sigma$ are equal modulo variable renaming,

(c) $L \leftarrow \bar{N}^{\prime}$ is the only clause in $P_{j}$ whose head is unifiable with $L \sigma$,

then $P_{i+1}=\left(P_{i} \backslash(H \leftarrow \bar{M}, \bar{N})\right) \cup(H \leftarrow \bar{M}, L \sigma)$. The pair of time-stamps $\left\langle L \sigma_{\mathrm{unf}}, L \sigma_{\mathrm{fld}}\right\rangle$ is $\langle 0, i+1\rangle$. Besides, the pair $\left\langle M_{\mathrm{unf}}, M_{\mathrm{fld}}\right\rangle$ in $P_{i+1}$ is equal to $\left\langle M_{\mathrm{unf}}, M_{\mathrm{fld}}\right\rangle$ in $P_{i}$ for each $M \in \bar{M}$.

Rule 4 Deletion. If $S$ is the definition of the predicate $p$ in $P_{i}, p \notin \operatorname{Pred}_{\mathcal{L}}\left(P_{0}\right)$ and $p \notin \operatorname{Pred}_{\mathcal{L}}\left(P_{i} \backslash S\right)$, then $P_{i+1}=\left(P_{i} \backslash S\right)$.

Note that the above rules can be used only if the definition of the involved literals exists. The definition of every positive literal always exists, but this is not the case for negative literals.

Using the above set of rules, an unfold/fold transformation system that preserves the Clark-Kunen semantics was introduced in [12].

Theorem 2. [12] If $\left\langle P_{0}, \ldots, P_{n}\right\rangle$ is a transformation sequence that is obtained using the rules New Definition, Unfolding, Folding and Deletion with the following two restrictions for each $0 \leq i \leq n-1$

- Unfolding is applied at the step $i+1$ only if it is not self-unfolding and the definition of the unfolded literal is taken from $P_{i}$, 
- Folding is applied at the step $i+1$ only if the folder clause is taken from $P_{i}$ and is different from the folded one,

then $P_{0}$ and $P_{j}$ are equivalent for every $0 \leq j \leq n$.

Proof. A formal proof of this result can be found in [12]. In fact, the authors provide a stronger result there since they prove the preservation of equivalence w.r.t. completion semantics. In particular, the rules New Definition and Deletion are correct since the Clark-Kunen semantics and completion semantics are relevant.

In the above unfold/fold transformation system, self-unfolding is not allowed. Next, we show that it is possible to prove the correctness w.r.t. the Clark-Kunen semantics if we allow self-unfolding. However, it is well known that self-unfolding does not preserve completion semantics (see [19]); that is, the logical equivalence between programs' completion.

Lemma 1. Let $\left\langle P_{0}, \ldots, P_{i}\right\rangle$ be a correct transformation sequence. If the program $P_{i+1}$ is obtained by self-unfolding, then $P_{i+1} \equiv P_{j}$ for every $1 \leq j \leq i$.

Proof. Since $P_{i}$ and $P_{j}$ are equivalent for every $1 \leq j \leq i$, it suffices to prove that $P_{i}$ and $P_{i+1}$ are equivalent. For this purpose and according to the stated notion of equivalence, we have to prove that $P_{i} \preceq P_{i+1}$ and $P_{i+1} \preceq P_{i}$. Let us assume that the unfolded literal $L$ occurs in a clause

$$
C=H \leftarrow \bar{M}, L
$$

where $\bar{z}=\operatorname{Var}(H \cdot \bar{M} \cdot L)$ and that the definition of $L$ in $P_{i}$ is given by

$$
\operatorname{Def}_{P_{i}}[L]=C \cup\left\langle L_{k} \leftarrow \bar{N}^{k} \mid 1 \leq k \leq m\right\rangle
$$

where $\bar{y}^{k}=\operatorname{Var}\left(L_{k} \cdot \bar{N}^{k}\right)$ for each $1 \leq k \leq m$. Without loss of generality, we also assume that $\operatorname{Def}_{P_{i}}[H]$ exclusively consists of the clause $C$. Hence, the definition of $H$ in the program $P_{i}$ is

$$
\operatorname{Def}_{P_{i+1}}[H]=\left\langle\left(H \leftarrow \bar{M}, \bar{N}^{k}\right) \theta_{k} \mid 1 \leq k \leq m\right\rangle
$$

where $\theta_{k}=\operatorname{mgu}\left(H, L_{k}\right)$ and $\bar{z}$ is renamed as $\bar{z}^{k}$ in the $k$-th clause for each $1 \leq k \leq m$.

Then, we first prove that $P_{i} \preceq P_{i+1}$. That is, we prove that for all $0 \in\{\mathrm{T}, \mathrm{F}\}$, $p \in\left(\operatorname{Pred}_{\mathcal{L}}\left(P_{i}\right) \cap \operatorname{Pred}_{\mathcal{L}}\left(P_{i+1}\right)\right)$ and $n \in \mathbb{N}$ there exists some $n^{\prime} \in \mathbb{N}$ such that

$$
\operatorname{FET}_{\mathcal{L}}=\left(\mathrm{O}_{n}^{P_{i}}[p(\bar{x})] \rightarrow \mathrm{O}_{n^{\prime}}^{P_{i+1}}[p(\bar{x})]\right)^{\forall} .
$$

The proof for operator $\mathrm{F}$ is constructed by induction on $n$. The case $n=0$ is trivial since the operator $\mathrm{F}$ always returns false at the first iteration. Assuming that the hypothesis holds for $n$, we prove the case for $n+1$. The proof for every 
literal that is not an instance of $H$ directly follows from the induction hypothesis, since $P_{i} \backslash \operatorname{Def}_{P_{i}}[H]=P_{i+1} \backslash \operatorname{Def}_{P_{i+1}}[H]$. Regarding $H$, the proof is

$$
\begin{array}{rlr}
\mathrm{F}_{n+1}^{P_{i}}[H] & =\forall \bar{z}\left(\mathrm{~F}_{n}^{P_{i}}[\bar{M}] \vee \mathrm{F}_{n}^{P_{i}}[L] \vee \neg \alpha\right) & \left(\text { by } \operatorname{Def}_{P_{i}}[H]\right) \\
& =\bigwedge_{k=1}^{m} \forall \bar{z}^{k} \cdot \bar{y}^{k}\left(\mathrm{~F}_{n}^{P_{i}}[\bar{M}] \vee \mathrm{F}_{n-1}^{P_{i}}\left[\bar{N}^{k}\right] \vee \neg \theta_{k}\right) & \text { (by } \left.\operatorname{Def}_{P_{i}}[L]\right) \\
& \rightarrow \bigwedge_{k=1}^{m} \forall \bar{z}^{k} \cdot \bar{y}^{k}\left(\mathrm{~F}_{n_{1}}^{P_{i+1}}[\bar{M}] \vee \mathrm{F}_{n_{1}}^{P_{i+1}}\left[\bar{N}^{k}\right] \vee \neg \theta_{k}\right) & \text { (by the induction } \\
& \text { hypothesis and Prop. 1) } \\
& =\mathrm{F}_{n^{\prime}}^{P_{i+1}}[H] & \text { (by Def } \left.P_{P_{i+1}}[H]\right)
\end{array}
$$

where $n^{\prime}=n_{1}+1$ and $\alpha$ is a renaming from the variables in $H$ to the variables in the head of $C$, since both literals are equal modulo variable renaming.

Second, we also prove that $P_{i+1} \preceq P_{i}$. In this case, we show that for all $0 \in\{\mathrm{T}, \mathrm{F}\}, p \in\left(\operatorname{Pred}_{\mathcal{L}}\left(P_{i}\right) \cap \operatorname{Pred}_{\mathcal{L}}\left(P_{i+1}\right)\right)$ and $n \in \mathbb{N}$ there exists some $n^{\prime \prime} \in \mathbb{N}$ such that

$$
\operatorname{FET}_{\mathcal{L}} \models\left(\mathrm{O}_{n}^{P_{i+1}}[p(\bar{x})] \rightarrow \mathrm{O}_{n^{\prime \prime}}^{P_{i}}[p(\bar{x})]\right)^{\forall}
$$

by induction on $n$. As before, the case $n=0$ is trivial and, assuming that the hypothesis holds for $n$, the case $n+1$ for any literal that is not an instance of $H$ directly follows from the induction hypothesis. Hence, focusing only on $H$, the proof is

$$
\begin{array}{rlr}
\mathrm{F}_{n+1}^{P_{i+1}}[H] & =\bigwedge_{k=1}^{m} \forall \bar{z}^{k} \cdot \bar{y}^{k}\left(\mathrm{~F}_{n}^{P_{i+1}}[\bar{M}] \vee \mathrm{F}_{n}^{P_{i+1}}\left[\bar{N}^{k}\right] \vee \neg \theta_{k}\right) & \text { (by Def } \left.P_{P_{i+1}}[H]\right) \\
& \rightarrow \bigwedge_{k=1}^{m} \forall \bar{z}^{k} \cdot \bar{y}^{k}\left(\mathrm{~F}_{n_{2}}^{P_{i}}[\bar{M}] \vee \mathrm{F}_{n_{2}}^{P_{i}}\left[\bar{N}^{k}\right] \vee \neg \theta_{k}\right) & \text { (by the induction } \\
& =\forall \bar{z}\left(\mathrm{~F}_{n_{2}}^{P_{i}}[\bar{M}] \vee \mathrm{F}_{n_{2}+1}^{P_{i}}[L] \vee \neg \alpha\right) & \text { hypothesis) } \\
& \left.\rightarrow \mathrm{F}_{n^{\prime \prime}}^{P_{i}}[H] \quad \text { (by Def } P_{i}[L]\right)
\end{array}
$$

where $n^{\prime \prime}=n_{2}+2$ and $\alpha$ is a renaming from $\operatorname{Var}(H)$ to $\operatorname{Var}(\operatorname{head}(C))$.

The next theorem is a direct consequence of Theorem 2 and Lemma 1.

Theorem 3. If $\left\langle P_{0}, \ldots, P_{n}\right\rangle$ is a transformation sequence that is obtained using the rules New Definition, Unfolding, Folding and Deletion with the following two restrictions for each $0 \leq i \leq n-1$

- Unfolding is applied at the step $i+1$ only if the definition of the unfolded literal is taken from $P_{i}$,

- Folding is applied at the step $i+1$ only if the folder clause is taken from $P_{i}$ and is different from the folded one,

then $P_{0}$ and $P_{j}$ are equivalent for every $0 \leq j \leq n$.

However, Example 2 shows a natural way for obtaining a recursive definition that cannot be obtained by the system described in Theorem 3 . 


\section{Generalized Folding}

In this section, we introduce less restrictive conditions for the rule Folding than the ones in Theorem 3. Our main aim is twofold. First, we will allow the folder clause to be taken from any program in the transformation sequence $\left\langle P_{0}, \ldots, P_{i}\right\rangle$. Second, we relax the requirement that every folded literal should come from unfolding. In our proposal, this condition is combined with a nonfailure requirement of the literals that do not come from unfolding.

If the folder clause comes from the actual program $P_{i}$, then Theorem 3 only requires the folder and the folded clause to be different, because the so-called self-folding is clearly incorrect. Note that the result of folding a clause $p \leftarrow r$ with itself is $p \leftarrow p$. Besides, when the folder clause could come from a program $P_{j}$ where $0 \leq j<i$, the self-folding transformation sometimes involves several clauses, which makes difficult to detect it. As a consequence, applicability conditions must be carefully designed to avoid problems related to the self-folding. The following example tries to illustrate this kind of problems.

Example 7. Let us consider the following transformation sequence.
$P_{0}:$
1. $p \leftarrow r$
2. $q \leftarrow r$
3. $r \longleftarrow$
(by folding $r$ in the clause 1 using the clause 2 of $P_{0}$ )
$P_{1}$ :
4. $p \leftarrow q$
2. $q \leftarrow r$
3. $r \leftarrow$
(by folding $r$ in the clause 2 using the clause 1 of $P_{0}$ )
$P_{2}$ :
4. $p \leftarrow q$
5. $q \leftarrow p$
3. $r \leftarrow$.

The first two programs are trivially equivalent. However, the goal $\leftarrow p$ loops in $P_{2}$, whereas it succeeds in the programs $P_{0}$ and $P_{1}$.

In order to prove that a transformation rule preserves equivalence we have to ensure that $P_{i+1} \preceq P_{i}$ and $P_{i} \preceq P_{i+1}$. In Theorem 4 , we show that $P_{i+1} \preceq P_{i}$ holds whenever $P_{i+1}$ is obtained by Folding from $P_{i}$.

Theorem 4. Let $\left\langle P_{0}, \ldots, P_{i}\right\rangle$ be a correct transformation sequence. If the program $P_{i+1}$ is obtained by the rule Folding, then $P_{i+1} \preceq P_{j}$ for every $1 \leq j \leq i$.

Proof. Assume that we fold the clause

$$
C=H \leftarrow \bar{M}, \bar{N}
$$

in the program $P_{i}$ using the clause

$$
L \leftarrow \bar{N}^{\prime}
$$

in a program $P_{j}$ for some $1 \leq j \leq i$, where $\operatorname{Def}_{P_{i}}[H]=C \cup\left\langle H_{k} \leftarrow \bar{B}^{k}\right| 2 \leq$ $k \leq m\rangle, \bar{z}^{1}=\operatorname{Var}(C)$ and $\bar{z}^{k}=\operatorname{Var}\left(H_{k} \cdot \bar{B}^{k}\right)$ for each $2 \leq k \leq m$. Hence, $P_{i+1}$ consists of the clauses

$$
P_{i+1}=\left(P_{i} \backslash C\right) \cup(H \leftarrow \bar{M}, L \sigma)
$$


where domain $(\sigma)=\operatorname{Var}(L), \bar{N}=\bar{N}^{\prime} \sigma, \bar{w}=\operatorname{Var}(H \cdot \bar{M} \cdot L \sigma)$ and $\bar{y}=\bar{z}^{1} \backslash \bar{w}$. Since $\left\langle P_{0}, \ldots, P_{i}\right\rangle$ is a correct transformation sequence, then $P_{i} \equiv P_{j}$ for every $1 \leq j \leq i$. Thus, it suffices to show that $P_{i+1} \preceq P_{i}$. According to Corollary 1 , we have to prove that for all $0 \in\{\mathrm{T}, \mathrm{F}\}, p \in\left(\operatorname{Pred}_{\mathcal{L}}\left(P_{i}\right) \cap \operatorname{Pred}_{\mathcal{L}}\left(P_{i+1}\right)\right)$ and $n \in \mathbb{N}$ there exists some $n^{\prime} \in \mathbb{N}$ such that

$$
\operatorname{FET}_{\mathcal{L}} \models\left(\mathrm{O}_{n}^{P_{i+1}}[p(\bar{x})] \rightarrow \mathrm{O}_{n^{\prime}}^{P_{i}}[p(\bar{x})]\right)^{\forall} .
$$

The proof (for the operator $\mathrm{F}$ ) is obtained by induction on $n$. The case $n=0$ is trivial because the operator $\mathrm{F}$ always returns false at the first iteration. Assuming that the hypothesis holds for $n$, the we prove the case for $n+1$. The proof directly follows from the induction hypothesis for every literal that is not an instance of $H$. Regarding $H$, the proof starts as follows

$$
\begin{aligned}
\mathrm{F}_{n+1}^{P_{i+1}}[H]= & \forall \bar{z}^{1}\left(\mathrm{~F}_{n}^{P_{i+1}}[\bar{M}] \vee \mathrm{F}_{n}^{P_{i+1}}[L \sigma] \vee \neg \alpha_{1}\right) \wedge \\
& \left.\bigwedge_{k=2}^{m} \forall \bar{z}^{k}\left(\mathrm{~F}_{n}^{P_{i+1}}\left[\bar{B}^{k}\right] \vee \neg \alpha_{k}\right) \quad \quad \text { (by } \operatorname{Def}_{P_{i+1}}[H]\right) \\
\rightarrow & \forall \bar{z}^{1}\left(\mathrm{~F}_{n_{1}}^{P_{i}}[\bar{M}] \vee \mathrm{F}_{n_{1}}^{P_{i}}[L \sigma] \vee \neg \alpha_{1}\right) \wedge \\
& \bigwedge_{k=2}^{m} \forall \bar{z}^{k}\left(\mathrm{~F}_{n_{1}}^{P_{i}}\left[\bar{B}^{k}\right] \vee \neg \alpha_{k}\right) \quad \text { (by the induction hypothesis) }
\end{aligned}
$$

where $\alpha_{k}=\operatorname{mgu}\left(H, H_{k}\right)$ for $2 \leq k \leq m$ and $\alpha_{1}$ is a renaming from the variables in $H$ to the variables in the head of $C$. Since $\left(L \leftarrow \bar{N}^{\prime}\right) \in P_{j}$, we know that

$$
\operatorname{FET}_{\mathcal{L}} \models\left(\mathrm{F}_{n+1}^{P_{j}}[L \sigma] \rightarrow \forall \bar{y}\left(\mathrm{~F}_{n}^{P_{j}}\left[\bar{N}^{\prime} \sigma\right]\right)\right)^{\forall}
$$

holds for all $n \in \mathbb{N}$. Further, we have that $P_{j} \equiv P_{i}$, thus for all $n \in \mathbb{N}$ there exists some $n^{\prime \prime} \in \mathbb{N}$ such that

$$
\operatorname{FET}_{\mathcal{L}} \models_{3}\left(\mathrm{~F}_{n}^{P_{i}}[L \sigma] \rightarrow \forall \bar{y}\left(\mathrm{~F}_{n^{\prime \prime}}^{P_{i}}\left[\bar{N}^{\prime} \sigma\right]\right)\right)^{\forall} .
$$

Hence, the proof continues as follows.

$$
\begin{aligned}
\mathrm{F}_{n+1}^{P_{i+1}}[H] \rightarrow & \forall \bar{z}^{1} \cdot \bar{y}\left(\mathrm{~F}_{n_{1}}^{P_{i}}[\bar{M}] \vee \mathrm{F}_{n_{2}}^{P_{i}}\left[\bar{N}^{\prime} \sigma\right] \vee \neg \alpha_{1}\right) \wedge \\
& \bigwedge_{k=2}^{m} \forall \bar{z}^{k}\left(\mathrm{~F}_{n_{1}}^{P_{i}}\left[\bar{B}^{k}\right] \vee \neg \alpha_{k}\right) \\
\rightarrow & \forall \bar{z}^{1} \cdot \bar{y}\left(\mathrm{~F}_{n_{3}}^{P_{i}}[\bar{M}] \vee \mathrm{F}_{n_{3}}^{P_{i}}\left[\bar{N}^{\prime} \sigma\right] \vee \neg \alpha_{1}\right) \wedge \\
& \bigwedge_{k=2}^{m} \forall \bar{z}^{k}\left(\mathrm{~F}_{n_{3}}^{P_{i}}\left[\bar{B}^{k}\right] \vee \neg \alpha_{k}\right) \\
= & \mathrm{F}_{n_{3}+1}^{P_{i}}[H]
\end{aligned}
$$

where $n_{3}=\max \left\{n_{1}, n_{2}\right\}$. Therefore, $P_{i+1} \preceq P_{i}$ and, by extension, $P_{i+1} \preceq P_{j}$ for every $1 \leq j \leq n$. 
However, when allowing use of a folder clause from any program in the transformation sequence, additional conditions are necessary in order to accomplish that $P_{i} \preceq P_{i+1}$. We formulate (in Theorem 5) side conditions for the Folding rule that depend on the literal that is introduced by Folding. To that end, we first introduce the following notion of fold-partitioned goals and then provide two auxiliary results.

Definition 4. Let $\left\langle P_{0}, \ldots, P_{j}, \ldots, P_{i}\right\rangle$ be a transformation sequence and $(H \leftarrow \bar{M}, \bar{N}) \in P_{i},\left(L \leftarrow \bar{N}^{\prime}\right) \in P_{j}$ be two clauses such that $\bar{N}=\bar{N}^{\prime} \sigma$. The goals $\leftarrow \bar{N}$ and $\leftarrow \bar{N}^{\prime}$ are fold-partitioned by $j$ into $\leftarrow \bar{A}, \bar{B}$ and $\leftarrow \bar{A}^{\prime}, \bar{B}^{\prime}$ iff

$-N_{\text {fld }} \leq j$ for every $N \in \bar{N}$,

- $B_{\text {unf }}>j$ for every $B \in \bar{B}$,

- no literal in $\bar{B}^{\prime}$ depends on $L$ in $P_{j}$.

Lemma 2. Let $\left\langle P_{0}, \ldots, P_{i}\right\rangle$ be a correct transformation sequence. If the program $P_{i+1}$ is obtained using the rule Folding from the program $P_{i}$ and the introduced literal does not depend on the head of the folded clause in $P_{i}$, then $P_{i+1}$ and $P_{j}$ are equivalent for every $0 \leq j \leq i$.

Proof. Since the programs $P_{i}$ and $P_{j}$ are equivalent for every $0 \leq j \leq i$, then it suffices to prove that $P_{i+1} \equiv P_{i}$. Besides, by Theorem 4 , we have that $P_{i+1} \preceq P_{i}$. Hence, it remains to prove that $P_{i} \preceq P_{i+1}$. Let us assume that the program $P_{i+1}$ is obtained by folding the clause

$$
C=H \leftarrow \bar{M}, \bar{N}
$$

in the program $P_{i}$ using the clause

$$
L \leftarrow \bar{N}^{\prime}
$$

in a program $P_{h}$ for some $0 \leq h \leq i$, where domain $(\sigma)=\operatorname{Var}(L), \bar{N}=\bar{N}^{\prime} \sigma$, $\operatorname{Def}_{P_{i}}[H]=C \cup\left\langle H_{k} \leftarrow \bar{B}^{k} \mid 2 \leq k \leq m\right\rangle, \bar{z}^{1}=\operatorname{Var}(C), \bar{z}^{k}=\operatorname{Var}\left(H_{k} \cdot \bar{B}^{k}\right)$ for each $2 \leq k \leq m$ and $L \sigma$ does not depend on $H$ in the program $P_{i}$. Therefore, the program $P_{i+1}$ is given by

$$
P_{i+1}=\left(P_{i} \backslash C\right) \cup(H \leftarrow \bar{M}, L \sigma)
$$

where domain $(\sigma)=\operatorname{Var}(L), \bar{N}=\bar{N}^{\prime} \sigma, \bar{w}=\operatorname{Var}(H \cdot \bar{M} \cdot L \sigma)$ and $\bar{y}=\bar{z}^{1} \backslash \bar{w}$.

Since $\left\langle P_{0}, \ldots, P_{i}\right\rangle$ is a correct transformation sequence, we know that $P_{i} \equiv$ $P_{j}$ for every $1 \leq j \leq i$. Then, we have to prove that for all $0 \in\{\mathrm{T}, \mathrm{F}\}, p \in$ $\left(\operatorname{Pred}_{\mathcal{L}}\left(P_{i}\right) \cap \operatorname{Pred}_{\mathcal{L}}\left(P_{i+1}\right)\right)$ and $n \in \mathbb{N}$, there exists some $n^{\prime} \in \mathbb{N}$ such that

$$
\operatorname{FET}_{\mathcal{L}}=\left(\left(0_{n}^{P_{i}}[p(\bar{x})] \rightarrow 0_{n^{\prime}}^{P_{i+1}}[p(\bar{x})]\right)^{\forall} .\right.
$$

The above implication is the reverse of the implication in formula (2) of Theorem 4. Because of this, the proofs by induction on $n$ for the operator $\mathrm{F}$ of the formulas (4) and (2) follow the same steps, but in the reverse order. As in Theorem 4, 
it also follows from the induction hypothesis that for every $n \in \mathbb{N}$ there exists some $n^{\prime \prime} \in \mathbb{N}$ such that

$$
\operatorname{FET}_{\mathcal{L}}=\left(\forall \bar{y}\left(\mathrm{~F}_{n}^{P_{i+1}}[\bar{N}]\right) \rightarrow \mathrm{F}_{n^{\prime \prime}}^{P_{i+1}}[L \sigma]\right)^{\forall}
$$

(that is, the dual property of (3)), since $L \sigma$ does not depend on $H$ in $P_{i}$.

Lemma 3. Let $\left\langle P_{0}, \ldots, P_{i}\right\rangle$ be a correct transformation sequence and $P_{i+1}$ the program that is obtained by folding a (folded) clause

$$
C=H \leftarrow \bar{M}, \bar{N}
$$

in the program $P_{i}$ using the (folder) clause

$$
D=L \leftarrow \bar{N}^{\prime}
$$

in the program $P_{j}$ for some $0 \leq j<i$ such that

$-\bar{N}=\bar{N}^{\prime} \sigma$,

- $H$ and $L$ are unifiable,

- the goal $\leftarrow \bar{N}$ is fold-partitioned by $j$ into $\leftarrow \bar{A}, \bar{B}$.

For any $n \in \mathbb{N}$ there exists some $n^{\prime} \in \mathbb{N}$ such that

$$
\operatorname{FET}_{\mathcal{L}}=\left(\mathrm{F}_{n}^{P_{i+1}}[B] \rightarrow \mathrm{F}_{n^{\prime}}^{P_{i+1}}[L \sigma]\right)^{\forall} .
$$

Moreover, if $\bar{A}$ is non-failing w.r.t. $\operatorname{Var}(L \sigma)$, then for any $n \in \mathbb{N}$ there exists some $n^{\prime \prime} \in \mathbb{N}$ such that

$$
\operatorname{FET}_{\mathcal{L}} \models\left(0_{n}^{P_{i}}[L \sigma] \rightarrow 0_{n^{\prime \prime}}^{P_{i+1}}[L \sigma]\right)^{\forall} .
$$

Proof. By condition (c) of the rule Folding (that is, $D$ is the only clause whose head and $L \sigma$ unify), we know that $\operatorname{Def}_{P_{j}}[L \sigma]$ consists of the single clause $D$. Besides, since $H$ and $L$ are unifiable, we also know that the clause $C$ has been obtained from $D$ by Unfolding. Since the goal $\leftarrow \bar{N}$ is fold-partitioned by $j$ into $\leftarrow \bar{A}, \bar{B}$, the clause $D$ can be rewritten as $D=L \leftarrow \bar{A}^{\prime}, \bar{B}^{\prime}$. For the sake of simplicity, we consider that $\bar{B}$ (resp. $\bar{B}^{\prime}$ ) consists of a single literal $B$ (resp. $B^{\prime}$ ) and also that $C$ has been obtained by (exactly) one unfolding step from $D$. The extension to the general case (that is, tuples consisting of more than one literal and an arbitrary number of unfolding steps) is straightforward.

According to the above conditions, we have that $P_{j}=P_{i-1}$ and that the clause $C$ has been obtained by unfolding the literal $B^{\prime}$ in the clause $D=L \leftarrow$ $\bar{A}^{\prime}, B^{\prime}$, where $\bar{v}=\operatorname{Var}\left(L \cdot \bar{A}^{\prime} \cdot B^{\prime}\right)$. Without loss of generality, we assume that the definition of $B^{\prime}$ in $P_{i-1}$ is

$$
\begin{aligned}
\operatorname{Def}_{P_{i-1}}\left[B^{\prime}\right]= & \left(B_{1} \leftarrow \bar{M}^{1}, B_{0}\right) \cup \\
& \left\langle B_{k} \leftarrow \bar{M}^{k} \mid 2 \leq k \leq m\right\rangle
\end{aligned}
$$


where $\alpha_{k}=\operatorname{mgu}\left(B^{\prime}, B_{k}\right)$ for each $1 \leq k \leq m, \bar{z}^{1}=\operatorname{Var}\left(B_{1} \cdot \bar{M}^{1} \cdot B_{0}\right)$ and $\bar{z}^{k}=\operatorname{Var}\left(B_{k} \cdot \bar{M}^{k}\right)$ for each $2 \leq k \leq m$. Moreover, since $B^{\prime}$ does not depend on $L$ in $P_{j}, D$ is not in $\operatorname{Def}_{P_{i-1}}\left[B^{\prime}\right]$. Therefore, the definition of $L \sigma$ in $P_{i}$ is given by

$$
\begin{aligned}
\operatorname{Def}_{P_{i}}[L \sigma]= & \left(L \leftarrow \bar{M}^{1}, \bar{A}^{\prime}, B_{0}\right) \alpha_{1} \cup \\
& \left\langle\left(L \leftarrow \bar{A}^{\prime}, \bar{M}^{k}\right) \alpha_{k} \mid 2 \leq k \leq m\right\rangle
\end{aligned}
$$

where the tuple of variables $\bar{v}$ is renamed as $\bar{v}^{k}$ in the $k$-th clause of $\operatorname{Def}_{P_{i}}[L \sigma]$ for each $1 \leq k \leq m$. Without loss of generality, we assume that the first clause in $\operatorname{Def}_{P_{i}}[L \sigma]$ is the folded one and, hence

$$
C=(H \leftarrow \bar{M}, \bar{A}, B)=\left(L \leftarrow \bar{M}^{1}, \bar{A}^{\prime}, B_{0}\right) \alpha_{1} .
$$

Finally, we fold the clause $C$ using $D$, obtaining the following definition of $L \sigma$

$$
\begin{aligned}
\operatorname{Def}_{P_{i+1}}[L \sigma]= & (H \leftarrow \bar{M}, L \sigma) \cup \\
& \left\langle\left(L \leftarrow \bar{A}^{\prime}, \bar{M}^{k}\right) \alpha_{k} \mid 2 \leq k \leq m\right\rangle
\end{aligned}
$$

in the program $P_{i+1}$, where $\left(\bar{A}^{\prime} \cdot B^{\prime}\right) \sigma=\bar{A} \cdot B, \bar{w}=\operatorname{Var}(H \cdot \bar{M} \cdot \bar{L} \sigma)$ and $\bar{y}=\left(\bar{v}^{1} \cup \bar{z}^{1}\right) \backslash \bar{w}$.

Then, we first prove property (a). Since $B=B^{\prime} \sigma$, for any $n \in \mathbb{N}$

$$
\begin{aligned}
& \mathrm{F}_{n+1}^{P_{i+1}}[B]= \forall \bar{z}^{1}\left(\mathrm{~F}_{n}^{P_{i+1}}\left[\bar{M}^{1}\right] \vee \mathrm{F}_{n}^{P_{i+1}}\left[B_{0}\right] \vee \neg \theta_{1}\right) \wedge \\
& \bigwedge_{k=2}^{m} \forall \bar{z}^{k}\left(\mathrm{~F}_{n}^{P_{i+1}}\left[\bar{M}^{k}\right] \vee \neg \theta_{k}\right)
\end{aligned}
$$

where $\theta_{k}=\operatorname{mgu}\left(B^{\prime} \sigma, B_{k}\right)$ for each $1 \leq k \leq m$. Besides, since $\alpha_{k}=\operatorname{mgu}\left(B^{\prime}, B_{k}\right)$, each pair of literals $\left\langle B^{\prime} \sigma \theta_{k}, B_{k} \theta_{k}\right\rangle$ is necessarily an instance of $\left\langle B^{\prime} \alpha_{k}, B_{k} \alpha_{k}\right\rangle$. Therefore, if $\rho_{k}=\operatorname{mgu}\left(B^{\prime} \sigma, B_{k} \alpha_{k}\right)=\operatorname{mgu}\left(B^{\prime} \sigma, B^{\prime} \alpha_{k}\right)$ for every $1 \leq k \leq m$, then the substitution $\theta_{k}$ can be rewritten as the composition $\alpha_{k} \rho_{k}$ and, thus

$$
\begin{aligned}
\mathrm{F}_{n+1}^{P_{i+1}}[B]= & \forall \bar{z}^{1}\left(\mathrm{~F}_{n}^{P_{i+1}}\left[\bar{M}^{1}\right] \vee \mathrm{F}_{n}^{P_{i+1}}\left[B_{0}\right] \vee \neg \alpha_{1} \vee \neg \rho_{1}\right) \wedge \\
& \bigwedge_{k=2}^{m} \forall \bar{z}^{k}\left(\mathrm{~F}_{n}^{P_{i+1}}\left[\bar{M}^{k}\right] \vee \neg \alpha_{k} \vee \neg \rho_{k}\right) \\
= & \forall \bar{z}^{1}\left(\mathrm{~F}_{n}^{P_{i+1}}\left[\bar{M}^{1} \alpha_{1}\right] \vee \mathrm{F}_{n}^{P_{i+1}}\left[B_{0} \alpha_{1}\right] \vee \neg \rho_{1}\right) \wedge \\
& \bigwedge_{k=2}^{m} \forall \bar{z}^{k}\left(\mathrm{~F}_{n}^{P_{i+1}}\left[\bar{M}^{k}\right] \vee \neg \alpha_{k} \vee \neg \rho_{k}\right) \\
= & \forall \bar{z}^{1}\left(\mathrm{~F}_{n}^{P_{i+1}}\left[\bar{M}^{1} \alpha_{1}\right] \vee \mathrm{F}_{n}^{P_{i+1}}[B] \vee \neg \rho_{1}\right) \wedge \\
& \bigwedge_{k=2}^{m} \forall \bar{z}^{k}\left(\mathrm{~F}_{n}^{P_{i+1}}\left[\bar{M}^{k}\right] \vee \neg \alpha_{k} \vee \neg \rho_{k}\right) .
\end{aligned}
$$


Now, according to the definition of $L \sigma$, we have that for any $n \in \mathbb{N}$

$$
\begin{aligned}
\mathrm{F}_{n+1}^{P_{i+1}}[L \sigma]= & \forall \bar{w}\left(\mathrm{~F}_{n}^{P_{i+1}}[\bar{M}] \vee \mathrm{F}_{n}^{P_{i+1}}[L \sigma] \vee \neg \gamma_{1}\right) \wedge \\
& \bigwedge_{k=2}^{m} \forall \bar{v}^{k} \cdot \bar{z}^{k}\left(\mathrm{~F}_{n-1}^{P_{i+1}}\left[\bar{A}^{\prime} \alpha_{k}\right] \vee \mathrm{F}_{n-1}^{P_{i+1}}\left[\bar{M}^{k} \alpha_{k}\right] \vee \neg \gamma_{k}\right) \\
= & \forall \bar{w}\left(\mathrm{~F}_{n}^{P_{i+1}}[\bar{M}] \vee \mathrm{F}_{n}^{P_{i+1}}[L \sigma] \vee \neg \gamma_{1}\right) \wedge \\
& \bigwedge_{k=2}^{m} \forall \bar{v}^{k} \cdot \bar{z}^{k}\left(\mathrm{~F}_{n-1}^{P_{i+1}}\left[\bar{A}^{\prime}\right] \vee \mathrm{F}_{n-1}^{P_{i+1}}\left[\bar{M}^{k}\right] \vee \neg \alpha_{k} \vee \neg \gamma_{k}\right) \\
= & \forall \bar{w}\left(\mathrm{~F}_{n}^{P_{i+1}}\left[\bar{M}^{1} \alpha_{1}\right] \vee \mathrm{F}_{n}^{P_{i+1}}[L \sigma] \vee \neg \gamma_{1}\right) \wedge \\
& \bigwedge_{k=2}^{m} \forall \bar{v}^{k} \cdot \bar{z}^{k}\left(\mathrm{~F}_{n-1}^{P_{i+1}}\left[\bar{A}^{\prime}\right] \vee \mathrm{F}_{n-1}^{P_{i+1}}\left[\bar{M}^{k}\right] \vee \neg \alpha_{k} \vee \neg \gamma_{k}\right)
\end{aligned}
$$

where $\gamma_{k}=\operatorname{mgu}\left(L \sigma, L \alpha_{k}\right)$ for every $1 \leq k \leq m .{ }^{5}$ Hence, since $\rho_{k}=\operatorname{mgu}\left(B^{\prime} \sigma, B^{\prime} \alpha_{k}\right)$, each substitution $\gamma_{k}$ is identical to $\rho_{k}$ and the above formula remains

$$
\begin{aligned}
\mathrm{F}_{n+1}^{P_{i+1}}[L \sigma]= & \forall \bar{w}\left(\mathrm{~F}_{n}^{P_{i+1}}\left[\bar{M}^{1} \alpha_{1}\right] \vee \mathrm{F}_{n}^{P_{i+1}}[L \sigma] \vee \neg \rho_{1}\right) \wedge \\
& \bigwedge_{k=2}^{m} \forall \bar{v}^{k} \cdot \bar{z}^{k}\left(\mathrm{~F}_{n-1}^{P_{i+1}}\left[\bar{A}^{\prime}\right] \vee \mathrm{F}_{n-1}^{P_{i+1}}\left[\bar{M}^{k}\right] \vee \neg \alpha_{k} \vee \neg \rho_{k}\right) .
\end{aligned}
$$

Thus, by induction on $n$, it is easy to see that the $k$-th conjunct in $\mathrm{F}_{n+1}^{P_{i+1}}[B]$ trivially implies the $k$-th conjunct in $\mathrm{F}_{n+1}^{P_{i+1}}[L \sigma]$ for each $1 \leq k \leq m$. Therefore, property (a) holds.

Next, we prove property (b). A proof for the operator T can be found in [15], where the authors provide an unfold/fold transformation system that allows even more transformations than the system described in Theorem 5 and prove that their system is correct w.r.t. the least Herbrand model. Here, assuming that $\bar{A}$ is non-failing w.r.t. $\operatorname{Var}(L \sigma)$ and using property (a), we prove that property (b) also holds for the operator F. For this purpose, we prove that for all $p \in$ $\left(\operatorname{Pred}_{\mathcal{L}}\left(P_{i}\right) \cap \operatorname{Pred}_{\mathcal{L}}\left(P_{i+1}\right)\right)$ and $n \in \mathbb{N}$ there exists some $n^{\prime} \in \mathbb{N}$ such that

$$
\operatorname{FET}_{\mathcal{L}} \models\left(\mathrm{F}_{n}^{P_{i}}[p(\bar{x})] \rightarrow \mathrm{F}_{n^{\prime}}^{P_{i+1}}[p(\bar{x})]\right)^{\forall} .
$$

The proof is obtained by induction on $n$. The case $n=0$ is trivial since the operator F always returns false at the first iteration. Assuming that the hypothesis holds for $n$, we prove the case for $n+1$. The proof directly follows from the induction hypothesis for every literal that is not an instance of $L \sigma$, since $\left(P_{i} \backslash \operatorname{Def}_{P_{i}}[L \sigma]\right)=\left(P_{i+1} \backslash \operatorname{Def}_{P_{i+1}}[L \sigma]\right)$. Regarding to $L \sigma$, the proof is as follows

$$
\begin{aligned}
& \mathrm{F}_{n+1}^{P_{i}}[L \sigma]=\forall \bar{v}^{1} \cdot \bar{z}^{1}\left(\mathrm{~F}_{n}^{P_{i}}[\bar{M}] \vee \mathrm{F}_{n}^{P_{i}}[\bar{A}] \vee \mathrm{F}_{n}^{P_{i}}[B] \vee \neg \gamma_{1}\right) \wedge \\
& \bigwedge_{k=2}^{m} \forall \bar{v}^{k} \cdot \bar{z}^{k}\left(\mathrm{~F}_{n}^{P_{i}}\left[\bar{A}^{\prime}\right] \vee \mathrm{F}_{n}^{P_{i}}\left[\bar{M}^{k}\right] \vee \neg \gamma_{k}\right) \\
& \text { (by } \operatorname{Def}_{P_{i}}[L \sigma] \text { ) }
\end{aligned}
$$

\footnotetext{
$\overline{{ }^{5} \text { Recall that }} H=L \alpha_{1}$ by (5).
} 


$$
\begin{aligned}
& \rightarrow \forall \bar{v}^{1} \cdot \bar{z}^{1}\left(\mathrm{~F}_{n_{1}}^{P_{i+1}}[\bar{M}] \vee \mathrm{F}_{n_{1}}^{P_{i+1}}[\bar{A}] \vee \mathrm{F}_{n_{1}}^{P_{i+1}}[B] \vee \neg \gamma_{1}\right) \wedge \\
& \bigwedge_{k=2}^{m} \forall \bar{v}^{k} \cdot \bar{z}^{k}\left(\mathrm{~F}_{n_{1}}^{P_{i+1}}\left[\bar{A}^{\prime}\right] \vee \mathrm{F}_{n_{1}}^{P_{i+1}}\left[\bar{M}^{k}\right] \vee \neg \gamma_{k}\right) \quad \text { (by the induction } \\
& \rightarrow \forall \bar{v}^{1} \cdot \bar{z}^{1}\left(\mathrm{~F}_{n_{2}}^{P_{i+1}}[\bar{M}] \vee \mathrm{F}_{n_{2}}^{P_{i+1}}[\bar{A}] \vee \mathrm{F}_{n_{2}}^{P_{i+1}}[L \sigma] \vee \neg \gamma_{1}\right) \wedge \\
& \bigwedge_{k=2}^{m} \forall \bar{v}^{k} \cdot \bar{z}^{k}\left(\mathrm{~F}_{n_{2}}^{P_{i+1}}\left[\bar{A}^{\prime}\right] \vee \mathrm{F}_{n_{2}}^{P_{i+1}}\left[\bar{M}^{k}\right] \vee \neg \gamma_{k}\right) \quad \text { (by property (a) } \\
& =\forall \bar{w}\left(\mathrm{~F}_{n_{2}}^{P_{i+1}}[\bar{M}] \vee \forall \bar{y}\left(\mathrm{~F}_{n_{2}}^{P_{i+1}}[\bar{A}]\right) \vee \mathrm{F}_{n_{2}}^{P_{i+1}}[L \sigma] \vee \neg \gamma_{1}\right) \wedge \\
& \bigwedge_{k=2}^{m} \forall \bar{v}^{k} \cdot \bar{z}^{k}\left(\mathrm{~F}_{n_{2}}^{P_{i+1}}\left[\bar{A}^{\prime}\right] \vee \mathrm{F}_{n_{2}}^{P_{i+1}}\left[\bar{M}^{k}\right] \vee \neg \gamma_{k}\right) \\
& =\forall \bar{w}\left(\mathrm{~F}_{n_{2}}^{P_{i+1}}[\bar{M}] \vee \mathrm{F}_{n_{2}}^{P_{i+1}}[L \sigma] \vee \neg \gamma_{1}\right) \wedge \\
& \bigwedge_{k=2}^{m} \forall \bar{v}^{k} \cdot \bar{z}^{k}\left(\mathrm{~F}_{n_{2}}^{P_{i+1}}\left[\bar{A}^{\prime}\right] \vee \mathrm{F}_{n_{2}}^{P_{i+1}}\left[\bar{M}^{k}\right] \vee \neg \gamma_{k}\right) \quad(\bar{A} \text { is non-failing } \\
& =\mathrm{F}_{n^{\prime}}^{P_{i+1}}[L \sigma] \\
& \text { on } \operatorname{Var}(L \sigma)) \\
& \text { (by } \operatorname{Def}_{P_{i+1}}[L \sigma] \text { ) }
\end{aligned}
$$

where $n^{\prime}=n_{2}+1$ and $\gamma_{k}=\operatorname{mgu}\left(L \sigma, L \alpha_{k}\right)$ for each $1 \leq k \leq m$. Note that $\operatorname{Var}(\bar{A}) \subseteq(\operatorname{Var}(L \sigma) \cup \bar{y})$, that is, we have defined $\bar{y}$ as the tuple given by $\left(\bar{v}^{1} \cup\right.$ $\left.\bar{z}^{1}\right) \backslash \bar{w}=\operatorname{Var}(H \cdot \bar{M} \cdot \bar{A} \cdot \bar{B}) \backslash \operatorname{Var}(H \cdot \bar{M} \cdot L \sigma)$ and, hence, we can define $\bar{y}$ as $\operatorname{Var}(\bar{A} \cdot \bar{B}) \backslash \operatorname{Var}(L \sigma)$, which trivially implies $\operatorname{Var}(\bar{A}) \subseteq(\operatorname{Var}(L \sigma) \cup \bar{y})$.

Now, we can formulate the side conditions for Folding in Theorem 5.

Theorem 5. If $\left\langle P_{0}, \ldots, P_{n}\right\rangle$ is a transformation sequence that is obtained using the rules New Definition, Unfolding, Folding and Deletion with the following restrictions for each $0 \leq i \leq n-1$

- Unfolding is applied at step $i+1$ only if the definition of the unfolded literal is taken from $P_{i}$.

- Folding is applied at the step $i+1$ if the folded clause $(H \leftarrow \bar{M}, \bar{N}) \in P_{i}$ and the folder clause $\left(L \leftarrow \bar{N}^{\prime}\right) \in P_{j}$ such that $0 \leq j \leq i$ and $\sigma=\operatorname{mgu}\left(\bar{N}, \bar{N}^{\prime}\right)$ satisfies one of the following conditions:

(1) $i=j$ and the folded clause is different from the folder one.

(2) $i>j$ and the literal $L \sigma$ does not depend on $H$ in the program $P_{i}$.

(3) $i>j, H$ and $L$ are unifiable, $\leftarrow \bar{N}$ is fold-partitioned by $j$ into $\leftarrow \bar{A}, \bar{B}$ and $\bar{A}$ is non-failing on $\operatorname{Var}(L \sigma)$.

Then, $P_{0}$ and $P_{k}$ are equivalent for every $0 \leq k \leq n$.

Proof. This is a direct consequence of Lemmas 2 and 3.

In the above theorem, condition (1) is given by Theorem 3. In condition (2), the literal introduced by Folding does not depend on the head of the folded clause in the program $P_{i}$. Condition (2) is illustrated by means of the following example. 
Example 8. Given the following program $P_{1}$

1. $\operatorname{add}(0, n, n) \leftarrow$

2. $\operatorname{add}\left(s\left(n_{1}\right), n_{2}, s\left(n_{3}\right) \leftarrow \operatorname{add}\left(n_{1}, n_{2}, n_{3}\right)\right.$

3. $\operatorname{add} 3\left(n_{1}, n_{2}, n_{3}, n_{4}\right) \leftarrow \operatorname{add}\left(n_{1}, n_{2}, y\right), \operatorname{add}\left(y, n_{3}, n_{4}\right)$.

First, we unfold the literal $\operatorname{add}\left(n_{1}, n_{2}, y\right)$ in clause 3 , obtaining

4. $\quad \operatorname{add} 3\left(0, n_{2}, n_{3}, n_{4}\right) \leftarrow \operatorname{add}\left(n_{2}, n_{3}, n_{4}\right)$

5. $\operatorname{add} 3\left(s\left(n_{1}\right), n_{2}, n_{3}, n_{4}\right) \leftarrow \operatorname{add}\left(n_{1}, n_{2}, y\right), \operatorname{add}\left(s(y), n_{3}, n_{4}\right)$

and then we unfold the literal $\operatorname{add}\left(s(y), n_{3}, n_{4}\right)$ in clause 5 , which yields

6. $\quad \operatorname{add} 3\left(s\left(n_{1}\right), n_{2}, n_{3}, s\left(n_{4}\right)\right) \leftarrow \operatorname{add}\left(n_{1}, n_{2}, y\right), \operatorname{add}\left(y, n_{3}, n_{4}\right)$.

Second, we fold the literals $\left\langle\operatorname{add}\left(n_{1}, n_{2}, y\right)\right.$, add $\left.\left(y, n_{3}, n_{4}\right)\right\rangle$ in clause 6 using clause 3 . Note that this transformation preserves equivalence according to Condition 3 , since both literals have been obtained by Unfolding and, hence, $\bar{A}$ denotes the empty tuple. The resulting clause is

7. $\operatorname{add} 3\left(s\left(n_{1}\right), n_{2}, n_{3}, s\left(n_{4}\right)\right) \leftarrow \operatorname{add} 3\left(n_{1}, n_{2}, n_{3}, n_{4}\right)$

in the program $P_{2}=\langle 1,2,4,7\rangle$. Third, we introduce a new predicate $a d d 4 / 5$ defined by the single clause

8. $\operatorname{add} 4\left(n_{1}, n_{2}, n_{3}, n_{4}, n_{5}\right) \leftarrow \operatorname{add}\left(n_{1}, n_{2}, y_{1}\right), \operatorname{add}\left(y_{1}, n_{3}, y_{2}\right)$, $\operatorname{add}\left(y_{2}, n_{4}, n_{5}\right)$

and obtain the program $P_{3}=P_{2} \cup\{8\}$. By means of Condition (2), the body literals $\left\langle\operatorname{add}\left(n_{1}, n_{2}, y_{1}\right)\right.$, add $\left.\left(y_{1}, n_{3}, y_{2}\right)\right\rangle$ in clause 8 can be folded using clause 3 in the program $P_{1}$ and the resulting literal is $a d d 3\left(n_{1}, n_{2}, n_{3}, y_{2}\right)$, which does not depend on $\operatorname{add} 4\left(n_{1}, n_{2}, n_{3}, n_{4}, n_{5}\right)$ in the program $P_{3}$. Hence, the final program is

1. $\quad \operatorname{add}(0, n, n) \leftarrow$

2. $\quad \operatorname{add}\left(s\left(n_{1}\right), n_{2}, s\left(n_{3}\right) \leftarrow \operatorname{add}\left(n_{1}, n_{2}, n_{3}\right)\right.$

4. $\operatorname{add} 3\left(0, n_{2}, n_{3}, n_{4}\right) \leftarrow \operatorname{add}\left(n_{2}, n_{3}, n_{4}\right)$

7. $\quad \operatorname{add} 3\left(s\left(n_{1}\right), n_{2}, n_{3}, s\left(n_{4}\right)\right) \leftarrow \operatorname{add} 3\left(n_{1}, n_{2}, n_{3}, n_{4}\right)$

9. $\operatorname{add} 4\left(n_{1}, n_{2}, n_{3}, n_{4}, n_{5}\right) \leftarrow \operatorname{add} 3\left(n_{1}, n_{2}, n_{3}, y_{2}\right), \operatorname{add}\left(y_{2}, n_{4}, n_{5}\right)$.

It could be argued that there exists a reordering of the above transformation sequence in such a way that the system described in Theorem 3 allows to fold the literals $\left\langle\operatorname{add}\left(n_{1}, n_{2}, y_{1}\right)\right.$, add $\left.\left(y_{1}, n_{3}, y_{2}\right)\right\rangle$ in the definition of $\operatorname{add} 4_{/ 5}$ : in this case, it would be enough to introduce add4/5 and fold its body literals before transforming the definition of sum $3 / 4$. However, such a restriction in the order of rule application unnecessarily complicates some transformation sequences, which may involve a large number of clauses.

Regarding condition (3), it is worthwhile to remark that its combination with condition (c) of Folding (that is, the literal introduced by Folding only unifies with the head of the folder clause in the program $P_{j}$ ) ensures that the folded clause has been obtained exclusively by unfolding transformations from the folder 
clause. Otherwise, if the folded clause is not obtained from the folder one, then the introduced literal would unify with the head of at least two clauses in $P_{j}$. Condition (3) corresponds to Examples 2 and 7, where the new literal depends on the clause head in $P_{i}$. Note that Example 7 does not satisfy Condition 3 since $q$ and $p$ do not unify. Besides, as we have already mentioned in Example 8, if $\bar{A}$ is an empty tuple (that is, all the literals in the folded clause comes from Unfolding), then the Folding rule using condition (3) is very similar to the one in the proposals $[5,23]$, where the authors also require all the literals to come from an unfolding to allow folding.

Next, we show that the transformation in Example 2 can be performed using the system in Theorem 5.

Example 2 (Contd.). From the initial program $P_{0}=\langle 1,2,3\rangle$, we obtain $P_{1}=$ $\langle 2,3,4,5\rangle$ by unfolding member $\left(y, x_{1}\right)$ in clause 1 using the clauses 2 and 3 . Then, Theorem 5 allows the folding of the body

$$
\left\langle\operatorname{member}\left(w, z_{1}\right), \neg \operatorname{member}\left(w, z_{2}\right)\right\rangle
$$

of clause 5 using clause 1 by means of the third condition in Folding . First, the head of the folder and the folded clause, which are taken from different programs ( $P_{1}$ and $P_{0}$ respectively), unify. Second, the literal member $\left(w, z_{1}\right)$ has been obtained by unfolding from $P_{0}$. Finally, the literal $\neg$ member $\left(w, z_{2}\right)$, which has not been obtained by unfolding, is non-failing on $z_{2}{ }^{6}$ according to the definition of $\neg$ member in Example 3. That is, there always exists a value for $w$ such that the goal $\leftarrow \neg$ member $\left(w, z_{2}\right)$ does not fail.

Note that if the literal $\neg$ member $\left(w, z_{2}\right)$ were failing on $z_{2}$, then the goal $\leftarrow$ $q\left(x_{1}, x_{2}\right)$ would fail in $P_{0}$, whereas $q\left(x_{1}, x_{2}\right)$ could not fail in $P_{2}$. That is the case in Example 1, where the literal $r$ is failing and, thus, the goal $\leftarrow p$ fails in the program $P_{0}$ and cycles in $P_{2}$.

The following example shows a transformation using the system in Theorem 5 that is mentioned in [25] as an example of unfeasible transformation under the system proposed in that paper.

Example 9. Let $\mathcal{F}_{\mathcal{L}}=\left\{a_{/ 0}, b_{/ 0}, c_{/ 0}\right\}$ and $P_{0}$ be the following program

1. $\operatorname{reach}(x, y) \leftarrow \operatorname{arc}(x, y)$

2. $\operatorname{reach}(x, y) \leftarrow \operatorname{arc}(x, w), \operatorname{reach}(w, y)$

3. $\quad \operatorname{br}(x, y, z) \leftarrow \operatorname{reach}(x, z), \operatorname{reach}(y, z)$

4. $\operatorname{arc}(a, b)$

5. $\operatorname{arc}(b, c)$

6. $\operatorname{arc}(c, a)$.

First, we unfold the literal reach $(x, z)$ in the clause 3 using the clauses 1 and 2 . The resulting program is $P_{1}=\langle 1,2,7,8,4,5,6\rangle$ where

7. $\operatorname{br}(x, y, z) \leftarrow \operatorname{arc}(x, z), \operatorname{reach}(y, z)$

8. $\operatorname{br}(x, y, z) \leftarrow \operatorname{arc}(x, w), \operatorname{reach}(w, z), \operatorname{reach}(y, z)$.

\footnotetext{
$\overline{{ }^{6} \text { The variable }} z_{1}$ from $q\left(z_{1}, z_{2}\right)$ is omitted since it does not occur in $\neg$ member $\left(w, z_{2}\right)$.
} 
Then, Theorem 5 allows the folding of literals $\langle\operatorname{reach}(w, z), \operatorname{reach}(y, z)\rangle$ in clause 8 using clause 3 and obtaining the literal $\operatorname{br}(w, y, z)$, since $\operatorname{reach}(w, z)$ has been obtained by unfolding from clause 3 and $\operatorname{reach}(y, z)$, which is inherited from clause 3 , is non-failing on $\langle y, z\rangle$. Note that $\operatorname{reach}(y, z)$ cannot fail since all the nodes $a, b$ and $c$ are reachable from any node. The resulting program is $P_{2}=$ $\langle 1,2,7,9,4,5,6\rangle$ where

9. $\quad \operatorname{br}(x, y, z) \leftarrow \operatorname{arc}(x, w), \operatorname{br}(w, y, z)$

which is equivalent to the programs $P_{0}$ and $P_{1}$.

Now, let us consider the program $P_{0}^{\prime}=\left\langle 1,2,3,4,5,6^{\prime}\right\rangle$ where

6'. $\operatorname{arc}(c, b)$.

As before, by unfolding reach $(x, z)$ in the clause 3 using the clauses 1 and 2 , we obtain the program $P_{1}^{\prime}=\left\langle 1,2,7,8,4,5,6^{\prime}\right\rangle$. However, we cannot fold $\langle\operatorname{reach}(w, z), \operatorname{reach}(y, z)\rangle$ in the clause 8 using the clause 3 since $\operatorname{reach}(y, z)$, which is inherited from $P_{0}^{\prime}$, is failing on $\langle y, z\rangle$; for example, $a$ is not reachable from $b$.

It is worth noting that the non-failing condition, which depends on the facts $\operatorname{arc}\left({ }_{-},-\right)$, makes the first transformation possible but not the second one. Thus, we allow only the transformations that are correct w.r.t. the graph. However, in [25] any transformation of this kind is forbidden irrespectively of the graph definition.

\section{Conclusions and Future Work}

We have introduced syntactic conditions for the rule Folding under which unfold/fold systems perform new kinds of transformations. In particular, the new conditions enable us to obtain recursive definitions and to remove local variables. This is possible because we allow the use of folder clauses from any program in the transformation sequence. The proposed transformation system is applicable to the whole class of normal logic programs and it is worth noting that only the negative literals without definition (due to the presence of local variables in the definition of its positive counterparts) cannot be used by Unfolding and Folding.

The need for providing new applicability conditions for the rule Folding has been motivated by means of some examples that show the risk of allowing transformations which use removed clauses. In this paper, we have concentrated on the rule Folding. However, similar problems arise in other transformation rules, such as Unfolding. For example, if we allowed unfolding by using definitions in previous programs, then the following transformation sequence could be obtained

$$
\begin{array}{ccccc}
P_{0}: & p \leftarrow q & q \leftarrow r & r & \leftarrow \\
& \text { (by unfolding } q \text { in the } 2^{\text {nd }} \text { clause using the definition in } P_{0} \text { ) } \\
P_{1}: & p \leftarrow r & q \leftarrow r & r \leftarrow \\
& \text { (by folding } h \text { in the } 3^{\text {rd }} \text { clause using the } 2^{\text {nd }} \text { clause) }
\end{array}
$$




$$
P_{2}: \quad p \leftarrow r \quad q \leftarrow p \quad r \leftarrow
$$

(by unfolding $p$ in the $3^{r d}$ clause using the definition in $P_{0}$ )

$$
P_{3}: \quad p \leftarrow r \quad q \leftarrow q \quad r \leftarrow
$$

Clearly, the last program is not equivalent to any of the previous ones (even w.r.t. the least Herbrand model), because the goal $\leftarrow q$ loops instead of succeeding. To find syntactic conditions that ensure correctness when using clauses from any program in the transformation sequence in other transformations rules (such as Unfolding, Replacement, etc.) is an interesting open problem.

\section{References}

1. J. Álvez, P. Lucio, F. Orejas, E. Pasarella, and E. Pino. Constructive negation by bottom-up computation of literal answers. In H. Haddad, A. Omicini, R. L. Wainwright, and L. M. Liebrock, editors, Proceedings of the 2004 ACM Symposium on Applied Computing (SAC), pages 1468-1475, 2004.

2. K. R. Apt. Logic programming. In Handbook of Theoretical Computer Science, Volume B: Formal Models and Sematics (B), pages 493-574. Elsevier, 1990.

3. R. Barbuti, P. Mancarella, D. Pedreschi, and F. Turini. A transformational approach to negation in logic programming. J. Log. Program., 8(3):201-228, 1990.

4. A. Bossi, N. Cocco, and S. Etalle. Simultaneous replacement in normal programs. J. Log. Comput., 6(1):79-120, 1996.

5. A. Bossi and S. Etalle. More on unfold/fold transformations of normal programs: Preservation of fitting's semantics. In L. Fribourg and F. Turini, editors, Logic Programming Synthesis and Transformation - Meta-Programming in Logic. 4th Internation Workshops, LOPSTR'94 and META'94, Pisa, Italy, June 20-21, 1994, volume 883 of Lecture Notes in Computer Science, pages 311-331. Springer, 1994.

6. R. M. Burstall and J. Darlington. A transformation system for developing recursive programs. J. ACM, 24(1):44-67, 1977.

7. D. Chan. Constructive negation based on the completed database. In R. A. Kowalski and K. A. Bowen, editors, Proceedings of the Fifth International Conference and Symposium on Logic Programming, pages 111-125. MIT Press, 1988.

8. K. L. Clark. Negation as failure. In H. Gallaire and J. Minker, editors, Logic and Data Bases, pages 293-322. Plenum Press, 1978.

9. H. Comon and P. Lescanne. Equational problems and disunification. J. Symb. Comput., 7(3/4):371-425, 1989.

10. S. K. Debray, P. López-García, and M. V. Hermenegildo. Non-failure analysis for logic programs. In L. Naish, editor, Logic Programming, Proceedings of the Fourteenth International Conference on Logic Programming, July 8-11, 1997, Leuven, Belgium, pages 48-62. MIT Press, 1997.

11. J. Dix. A classification theory of semantics of normal logic programs: II. weak properties. Fundam. Inform., 22(3):257-288, 1995.

12. P. A. Gardner and J. C. Shepherdson. Unfold/fold transformations of logic programs. In Computational Logic - Essays in Honor of Alan Robinson, pages 565-583, 1991.

13. A. V. Gelder, K. Ross, and J. S. Schlipf. Unfounded sets and well-founded semantics for general logic programs. In PODS '88: Proceedings of the seventh ACM SIGACT-SIGMOD-SIGART symposium on Principles of database systems, pages 221-230, New York, NY, USA, 1988. ACM Press. 
14. M. Gergatsoulis and M. Katzouraki. Unfold/fold transformations for definite clause programs. In M. V. Hermenegildo and J. Penjam, editors, Programming Language Implementation and Logic Programming, 6th International Symposium, PLILP'94, Madrid, Spain, September 14-16, 1994, volume 844 of Lecture Notes in Computer Science, pages 340-354, 1994.

15. T. Kanamori and H. Fujita. Unfold/fold transformation of logic programs with counters. Technical Report TR-179, ICOT Institute for New Generation Computer Technology, 1986.

16. K. Kunen. Negation in logic programming. J. Log. Program., 4(4):289-308, 1987.

17. J. W. Lloyd. Foundations of Logic Programming. Springer-Verlag, Berlin, second edition, 1987.

18. P. Lucio, F. Orejas, and E. Pino. An algebraic framework for the definition of compositional semantics of normal logic programs. J. Log. Program., 40(1):89$124,1999$.

19. M. J. Maher. Correctness of a logic program transformation system. Technical Report RC 13496, IBM T.J. Watson Research Center, 1988.

20. M. J. Maher. A tranformation system for deductive databases modules with perfect model semantics. Theor. Comput. Sci., 110(2):377-403, 1993.

21. A. Pettorossi and M. Proietti. Transformation of logic programs. In D. M. Gabbayand, C. J. Hogger, and J. A. Robinson, editors, Handbook of Logic in Artificial Intelligence and Logic Programming, Vol. 5, pages 697-787. Oxford University Press, 1998.

22. A. Roychoudhury, K. N. Kumar, C. R. Ramakrishnan, and I. V. Ramakrishnan. Beyond tamaki-sato style unfold/fold transformations for normal logic programs. Int. J. Found. Comput. Sci., 13(3):387-403, 2002.

23. T. Sato. Equivalence-preserving first-order unfold/fold transformation systems. Theor. Comput. Sci., 105(1):57-84, 1992.

24. T. Sato and H. Tamaki. Transformational logic program synthesis. In Proceedings of the International Conference on Fifth Generation Computer Systems, pages 195201, 1984.

25. H. Seki. Unfold/fold transformations of stratified programs. Theor. Comput. Sci., 86(1):107-139, 1991

26. H. Seki. Unfold/fold transformation of general logic programs for the well-founded semantics. J. Log. Program., 16(1):5-23, 1993.

27. J. C. Shepherdson. Language and equality theory in logic programming. Technical Report PM-91-02, University of Bristol, 1991.

28. H. Tamaki and T. Sato. Unfold/fold transformation of logic programs. In S.Å. Tärnlund, editor, Proceedings of the Second International Logic Programming Conference, Uppsala University, Uppsala, Sweden, pages 127-138, 1984. 